\title{
Simulación de la convección natural en un horno eléctrico
}

\section{Simulation of the natural convection in an electrical oven}

\author{
Goñi, Sandro M., (1) Salvadori, Viviana O. (1)
}

(1) Centro de Investigación y Desarrollo en Criotecnología de Alimentos (CIDCA) CCT-CONICET, Universidad Nacional de La Plata, Argentina - Facultad de Ingeniería, Universidad Nacional de La Plata, Argentina.

Contacto:smgoni@quimica.unlp.edu.ar

RECIBIDO: 29/2/2016 - APROBADO: 4/7/2016

\begin{abstract}
Resumen
Los productos cárnicos y panificados son de consumo masivo en numerosos países y suponen un consumo de energía elevado y, cada vez en mayor medida, el uso hornos eléctricos. En este sentido, es importante desarrollar hornos eficientes. El objetivo de este trabajo fue desarrollar un modelo preliminar de la fluido-dinámica por convección natural dentro de un horno eléctrico. Se utilizó un horno doméstico para los experimentos y dada la simetría del horno en las simulaciones numéricas se trabajó con un cuarto del volumen real. Para calcular el flujo de aire en el interior del horno se adoptó un modelo 3D de flujo laminar incompresible, acoplando el balance de energía, y la aproximación de Boussinesq para describir las fuerzas de flotación. En el balance de energía se impuso la temperatura en el techo y el piso, y en la pared y la puerta se estableció pérdida de energía por convección. El modelo se validó aceptablemente con determinaciones experimentales de perfiles de temperatura en diferentes posiciones del horno. Las variaciones de temperaturas observadas experimentalmente sugieren que el modelo laminar y la aproximación de Boussinesq están al límite de sus posibilidades, por lo cual se prevé en futuros trabajos usar modelos de turbulencia. Palabras clave: Consumo energético, hornos eléctricos, fluido-dinámica.
\end{abstract}

\begin{abstract}
Meat and bakery products are widely consumed in many countries and this process presents a high energy consumption. At the same time the use of electric ovens is increasing. In this regard, the development of efficient ovens becomes more important.

The aim of this work was to develop a preliminary model of the natural convection fluid-dynamics in an electric oven. A domestic oven was employed in the experiments and for simulation purposes a model of a quarter of its actual volume was employed. The air flow inside the oven was described using a laminar incompressible 3D model coupled to the energy balance, using the Boussinesq approximation to describe buoyancy forces. In the energy balance the temperature was imposed on the roof and the floor, whilst energy loss by convection was established at the side walls and the door. The model was reasonably validated with temperature profiles measured in different positions in the oven. The measured temperature variations indicate that the laminar model and the Boussinesq approximation are hardly applicable, then turbulence models will be used in future works.

Keywords: Energy consumption, electric ovens, fluid-dynamics.
\end{abstract}

\section{Introducción}

La cocción de alimentos en hornos es un proceso ampliamente utilizado en numerosos países, tanto a nivel hogareño como a nivel industrial. El horneado se aplica principalmente a productos panificados y cárnicos, los cuales son de consumo masivo. Bajo esta forma de cocción, los productos adquieren características organolépticas únicas y deseables, entre ellas la formación de una corteza superficial deshidratada característica y el desarrollo de sabores y aromas, las cuales no pueden ser obtenidas con ninguna otra técnica de cocción. A su vez, el horneado demanda un alto consumo energético (Goñi y Salvadori, 2012), debido a las altas temperaturas de operación empleadas (por lo general entre 180 y $250^{\circ} \mathrm{C}$ ). La energía consumida durante el proceso se divide en energía transferida al producto y pérdidas al ambiente. La energía transferida al producto se usa para su calentamiento, de manera tal de alcanzar algún valor de temperatura preestablecido, como una temperatura de seguridad microbiológica, $\mathrm{o}$ requerido por el proceso, como la temperatura de gelatinización del almidón en los productos panificados. $\mathrm{Al}$ mismo tiempo, una parte de la energía consumida por el producto se vincula a su deshidratación. En los alimentos panificados y cárnicos se verifica, por un lado, una elevada pérdida de peso debida a la evaporación de agua, lo que representa un alto consumo energético, pudiendo ser el calor latente mayor que el calor sensible intercambiado. Por otro lado, parte de la energía usada por el 
equipo se pierde hacia el ambiente, a través de las paredes del equipo y durante la apertura-cierre de la puerta. Estas pérdidas pueden llegar a ser considerables, de más del 50\% (Goñi y Salvadori, 2014). El uso de hornos eléctricos se está incrementando gradualmente, por diversos factores. En primer lugar, el gas natural o el gas licuado son combustibles no renovables, por lo cual se está tendiendo a disminuir su uso. Además, los hornos eléctricos tienen una gran variedad de tamaños (de unos 151 a más de $100 \mathrm{l}$ ), características (con o sin termostato, temporizador, selector de resistencias, potencias, spiedo, etcétera) y precios, son portátiles y se pueden usar en diferentes lugares, las instalaciones son considerablemente más simples, y para emprendimientos comerciales son más fáciles de implementar y habilitar, entre otros factores.

La tendencia mundial de clasificación, identificación y etiquetado de equipos en función de su eficiencia energética y/o de su consumo energético no se aplica a toda la gama de hornos disponibles. Solo el segmento más alto tiene algún tipo de clasificación energética. Considerando todos estos aspectos en forma conjunta, se percibe una oportunidad y una necesidad de realizar estudios de eficiencia energética de este tipo de equipos, tendiente a promover su clasificación energética. Esta información puede usarse de diferentes formas: para informar al consumidor, para informar a los organismos energéticos y de control estatales sobre el estado de situación, y, más importante, para asistir al Estado en la formulación de políticas o regulaciones tendientes a establecer estándares mínimos de eficiencia energética a los fabricantes e importadores de equipos.

Este trabajo se focaliza en el estudio de la fluido-dinámica dentro de los hornos, la cual es una valiosa herramienta para el estudio y diseño de hornos más eficientes (Mistry, et al., 2006, 2011; Khatir, et al., 2013). Desde hace varios años, el incremento de la capacidad de cálculo de las computadoras y el surgimiento de programas comerciales o gratuitos para resolver las ecuaciones de movimiento han favorecido este estudio (Norton, et al., 2006). Este ha sido abordado por diferentes autores que consideraron tanto únicamente la fluido-dinámica del horno como la interacción con un producto (Verboven, et al., 2000; Boulet, et al., 2010; Chhanwal, et al., 2010, 2011; Marra, et al., 2010; Smolka, et al., 2010; Paton, et al., 2013; Smolka, 2013; Ateeque, et al., 2014; Tzempelikos, et al., 2015).

En este trabajo se realizó un modelo simplificado preliminar de un horno eléctrico convectivo, usando un modo de cocción por convección natural. Se realizaron pruebas experimentales para la validación del modelo y los resultados obtenidos fueron satisfactorios.

\section{Materiales y Métodos}

\section{Determinaciones experimentales de temperatura}

Se utilizó un horno eléctrico de tipo doméstico de gama alta, marca ARISTON (FM87-FC, Italia). Este horno posee una cámara de cocción con longitudes efectivas de $0,435 \mathrm{~m}$ de ancho, $0,39 \mathrm{~m}$ de profundidad y $0,36 \mathrm{~m}$ de altura (Figura 1) y permite seleccionar uno entre siete modos de cocción. En este trabajo las experiencias se realizaron usando el modo de cocción convencional o natural, por medio del cual diferentes resistencias eléctricas calientan el aire de la cámara del horno y el movimiento del aire se produce por convección natural, debido a diferencias de densidad causadas por el calentamiento.

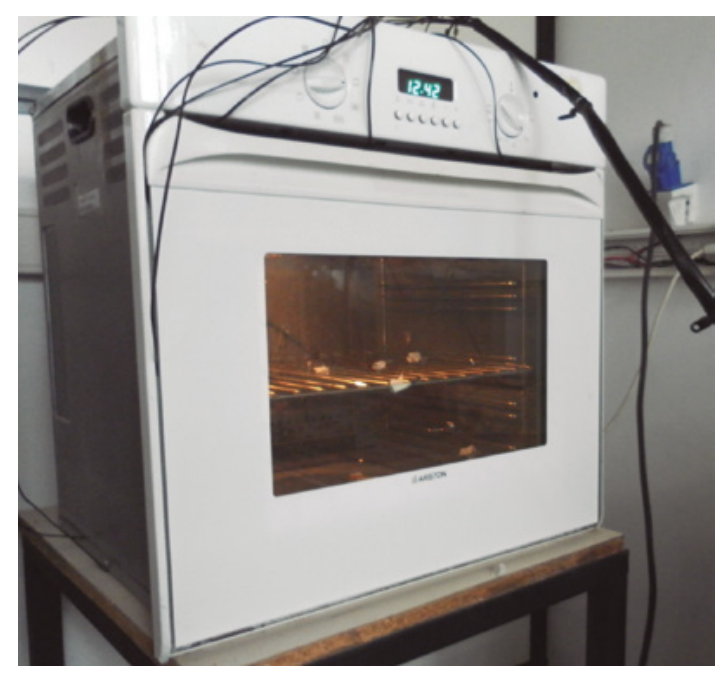

Figura 1. Horno eléctrico utilizado en las determinaciones experimentales, con las termocuplas colocadas.

Se midió la temperatura en diferentes posiciones del horno cada 5 seg utilizando termocuplas J y un adquisidor de datos FieldLogger (NOVUS Automation Inc., Brasil) conectado a una PC. Las determinaciones se realizaron partiendo desde un estado de reposo durante el tiempo suficiente para que se produzcan varios ciclos de actuación del sistema de control del horno en torno al valor de temperatura deseado. Cabe aclarar que se trabajó con el horno vacío, dado que el modelo a validar solo contempla los balances en el aire sin considerar la cocción o calentamiento de un alimento. Asimismo, se seleccionaron temperaturas bajas de manera tal de disminuir los aportes de energía por radiación, los cuales no fueron considerados en el modelo.

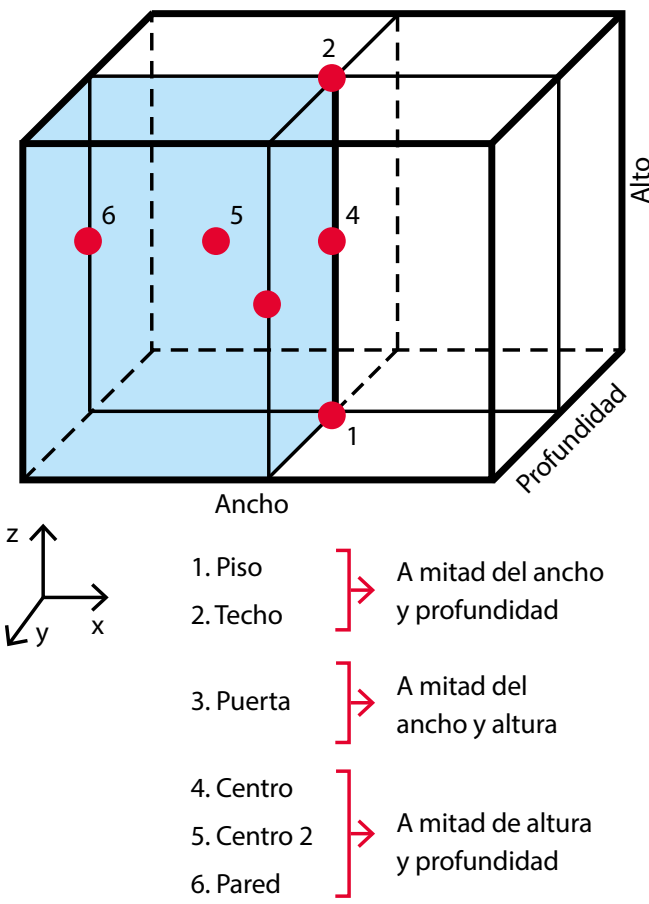

Figura 2. Representación esquemática de las posiciones de las termocuplas en la cámara del horno. Las termocuplas del piso (1) y del techo (2) se colocaron en contacto con las paredes; la termocupla de la pared (6) y de la puerta (3) se ubicaron a $5 \mathrm{~mm}$ de las respectivas superficies. 
Las posiciones de medición de temperatura se representan esquemáticamente en la Figura 2. No se realizaron mediciones de velocidad dado que no se dispone de equipamiento que soporte las temperaturas de operación empleadas en el horno. Adicionalmente, se realizaron medidas adicionales de temperatura en diferentes superficies internas del horno usando una cámara termográfica TESTO 875 (TESTO, Alemania).

\section{Modelado matemático}

Se consideró de forma preliminar que existen dos planos de simetría: uno perpendicular a la puerta (plano $y z$ ), a la mitad del ancho del horno, y el otro paralelo a la puerta (plano $x z$ ), a la mitad de la profundidad. Con estos supuestos se representó el horno usando una cuarta parte de su volumen. La aproximación final se observa en la Figura 3. En este trabajo preliminar no se incorporaron detalles de la geometría del horno, como resistencias, soporte de rejillas y bandejas, etcétera, debido a que aumentan considerablemente el costo computacional para resolver el modelo.

TECHO. Temperatura impuesta en el techo

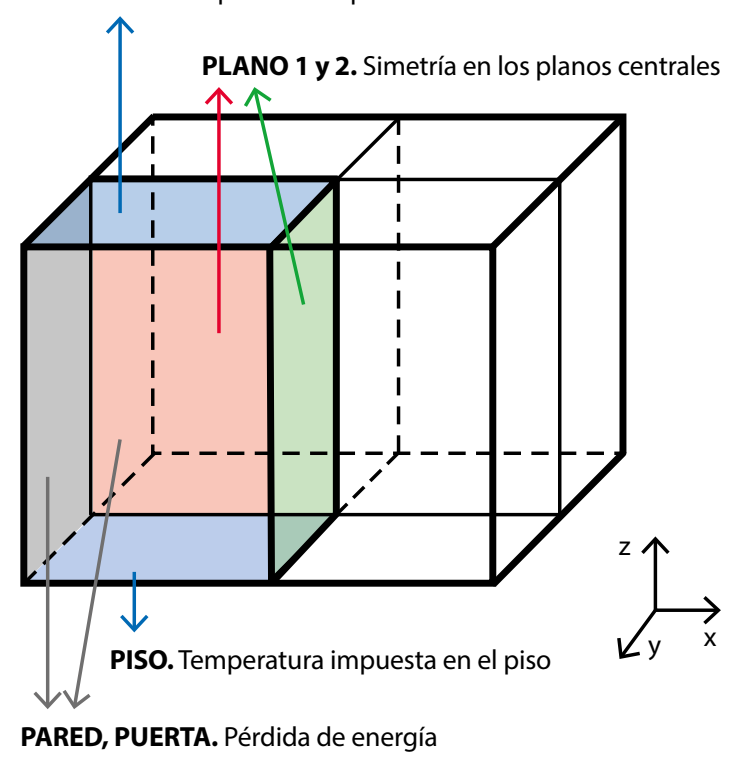

Figura 3. Aproximación tridimensional de la geometría del horno y detalles de sus fronteras y condiciones de contorno.

El movimiento del aire dentro del horno se obtiene resolviendo el balance de cantidad de movimiento, más conocido como ecuaciones de Navier-Stokes (Ec. (1)), dentro de la cámara del horno. En este trabajo se consideró que, dadas las pequeñas variaciones de presión en el interior del horno, puede considerarse al aire como un fluido incompresible; en consecuencia, no hay acumulación local de materia, lo que se describe mediante la ecuación de continuidad (Ec. (2)). En función del rango de velocidad del aire se adoptó régimen laminar. Más allá del supuesto de fluido incompresible, las variaciones de temperatura también afectan a la densidad del aire $y$, por ende, a las fuerzas de flotación, lo cual se puede modelar con diferentes grados de aproximación (Ferziger y Perić, 2002). Estas fuerzas, que determinan el movimiento del fluido en este sistema, se introdujeron usando la aproximación de Boussinesq (Ec. (3)), según la cual las fuerzas de flotación son proporcionales a la diferencia de temperatura respecto de un estado de referencia.

La variación local de temperatura se obtuvo resolviendo el balance de energía (Ec. (4)) en conjunto con el balance de cantidad de movimiento.

$\rho \frac{\partial \vec{u}}{\partial t}+\rho \vec{u} \cdot \nabla \vec{u}=\nabla \cdot\left(\mu\left(\nabla \vec{u}+(\nabla \vec{u})^{T}\right)\right)-\nabla \cdot p+\vec{F}$

$\nabla \cdot \vec{u}=0$

$\vec{F}=\rho_{\text {Ref }} \vec{g}\left(\beta\left(T-T_{R e f}\right)-1\right)$

$\rho C_{P} \frac{\partial T}{\partial t}+\rho C_{P} \vec{u} \cdot \nabla T=\nabla \cdot(k \nabla T)$

donde $\rho$ es la densidad del aire, $\mu$ es la viscosidad, $\beta$ es el coeficiente de expansión volumétrica, $C_{P}$ es la capacidad calorífica, $k$ es la conductividad térmica, $\vec{u}$ es el vector velocidad en las tres direcciones, $\vec{u}=\left(v_{x}, v_{y}, v_{z}\right)$, y la fuerza solo actúa en dirección vertical, dado $\vec{g}=(0 ; 0 ; 9,8)$. Como temperatura de referencia $T_{R e f}$ se usó la temperatura inicial del sistema, por lo cual la fuerza de flotación es nula inicialmente, y la densidad de referencia $\rho_{\text {Ref }}$ se calculó a dicha temperatura.

Las condiciones de contorno para el balance de cantidad de movimiento se establecieron como (ver nombres de fronteras en la Figura 3):

Velocidad nula en el TECHO, PARED, PUERTA, PISO:

$\vec{u}=0$

Simetría en los planos centrales (PLANO 1 y 2 ), donde $\vec{n}$ es el vector normal exterior a la superficie:

$\vec{n} \cdot \vec{u}=0$

Las condiciones de contorno para el balance de energía se establecieron como:

Flujo de Energía en la PARED y la PUERTA. Se estableció pérdida de energía por convección al ambiente usando un coeficiente de transferencia de energía $h_{\text {Efectivo }}$ y $\vec{q}$ es el flujo total de energía. La temperatura ambiente $T_{A m b}$ se estableció igual a la temperatura inicial del sistema. Los valores de $h_{\text {Efectivo }}$ fueron diferentes para la puerta y la pared y el más alto fue para la puerta. Estos valores tuvieron en cuenta el material y espesor de la pared y la puerta y los coeficientes de transferencia para el exterior y el interior estimados a partir de correlaciones.

$\vec{n} \cdot \vec{q}=h_{\text {Efectivo }}\left(T-T_{A m b}\right)$

Simetría en los planos centrales (PLANO 1 y 2):

$\vec{n} \cdot \vec{q}=0$

Temperatura Impuesta en el TECHO y el PISO. La temperatura de estas fronteras se estableció con los valores obtenidos experimentalmente.

$T_{\text {Piso }}=T_{P}(t)$

$T_{\text {Techo }}=T_{T}(t)$ 
El modelo se implementó en el software COMSOL ${ }^{\mathrm{TM} M u l-}$ tiPhysics versión 3.2, el cual utiliza el método de elementos finitos. Se realizaron numerosas pruebas de mallado y estrategias de resolución para lograr resultados aceptables en tiempos de simulación relativamente bajos.

\section{Resultados y Discusión}

Antes de realizar las pruebas experimentales orientadas a validar el modelo matemático se realizaron numerosas simulaciones con el objetivo de verificar la factibilidad del esquema numérico planteado. Así, se adoptaron diferentes combinaciones de temperaturas del techo y del piso (iguales o diferentes, constantes o variables, etcétera), resistencias prendidas/apagadas (temperatura impuesta o flujo nulo), distintos valores del coeficiente efectivo de transferencia de energía, diferentes mallados, etcétera. El modelo pudo ser resuelto, con mayor o menor dificultad, en todos los casos.

La Figura 4 muestra los perfiles de temperatura experimentales obtenidos durante una prueba fijando $100^{\circ} \mathrm{C}$ en el control del horno. Como puede observarse, la temperatura en el piso del horno se incrementa rápidamente y sobrepasa los $220^{\circ} \mathrm{C}$, hasta que comienza a actuar el sistema de control del horno. Similarmente, la temperatura del techo también pasa los $100^{\circ} \mathrm{C}$, pero en menor medida. A tiempos largos la temperatura en el piso varió entre 120 y $160^{\circ} \mathrm{C}$, con periodos de aproximadamente 9 $\mathrm{min}$, mientras que la temperatura en el techo lo hizo entre 110 y $130^{\circ} \mathrm{C}$. Inicialmente, el sistema de control del horno mantiene las resistencias encendidas hasta que el sensor de temperatura del equipo, ubicado en la parte central trasera, alcanza la temperatura deseada. Este retardo produce que las zonas cercanas a las resistencias estén a mucha mayor temperatura que la deseada. Con la cámara del horno vacía, la circulación de aire es más alta, por lo cual se prevé que con bandejas, etcétera, el sensor tarde más en registrar la temperatura deseada. La temperatura experimental más baja fue la registrada en las cercanías de la puerta del horno, lo cual indica que es una de las posiciones por donde se pierde mayor cantidad de energía.

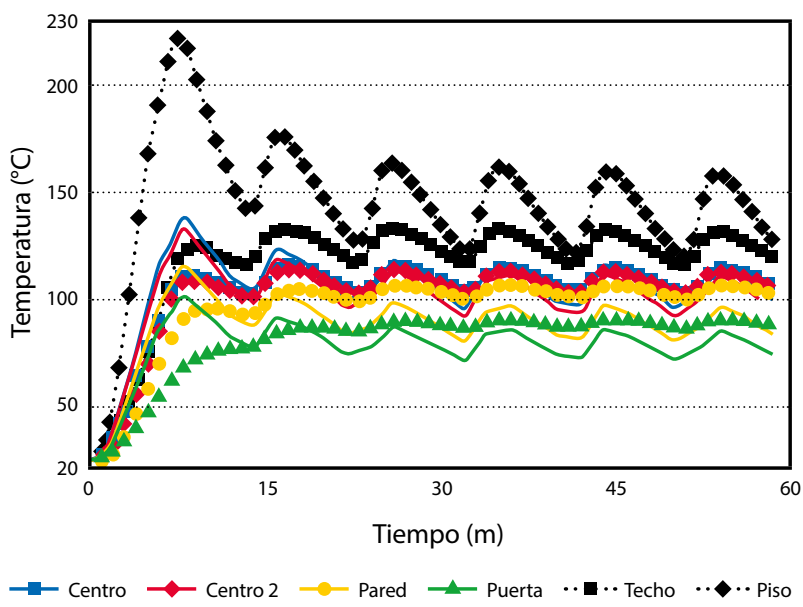

Figura 4. Variación de temperatura experimental (símbolos, cada 1 minuto) y simulada (líneas continuas) en diferentes posiciones del horno (dadas en diferentes colores). La temperatura simulada en el piso y el techo es igual que la experimental, dado que se usó condición de contorno prescripta.
El modelo final empleado para comparar con los resultados experimentales fue mallado con aproximadamente 115000 elementos tetraédricos (730000 incógnitas). El tiempo de simulación de 1 hora de proceso varió entre 2 y 3 $\mathrm{h}$, usando una PC de escritorio común (INTEL i5 2,67 GHz, 10 GB RAM). La frecuencia de variación de la temperatura simulada claramente coincide con la experimental, dado que se usaron estos perfiles en las condiciones de contorno del modelo. Para representar más adecuadamente el sistema se puede modelar también el sistema de control, de manera tal de poder usar el modelo sin la necesidad de contar con los perfiles experimentales a temperaturas arbitrarias.

a.

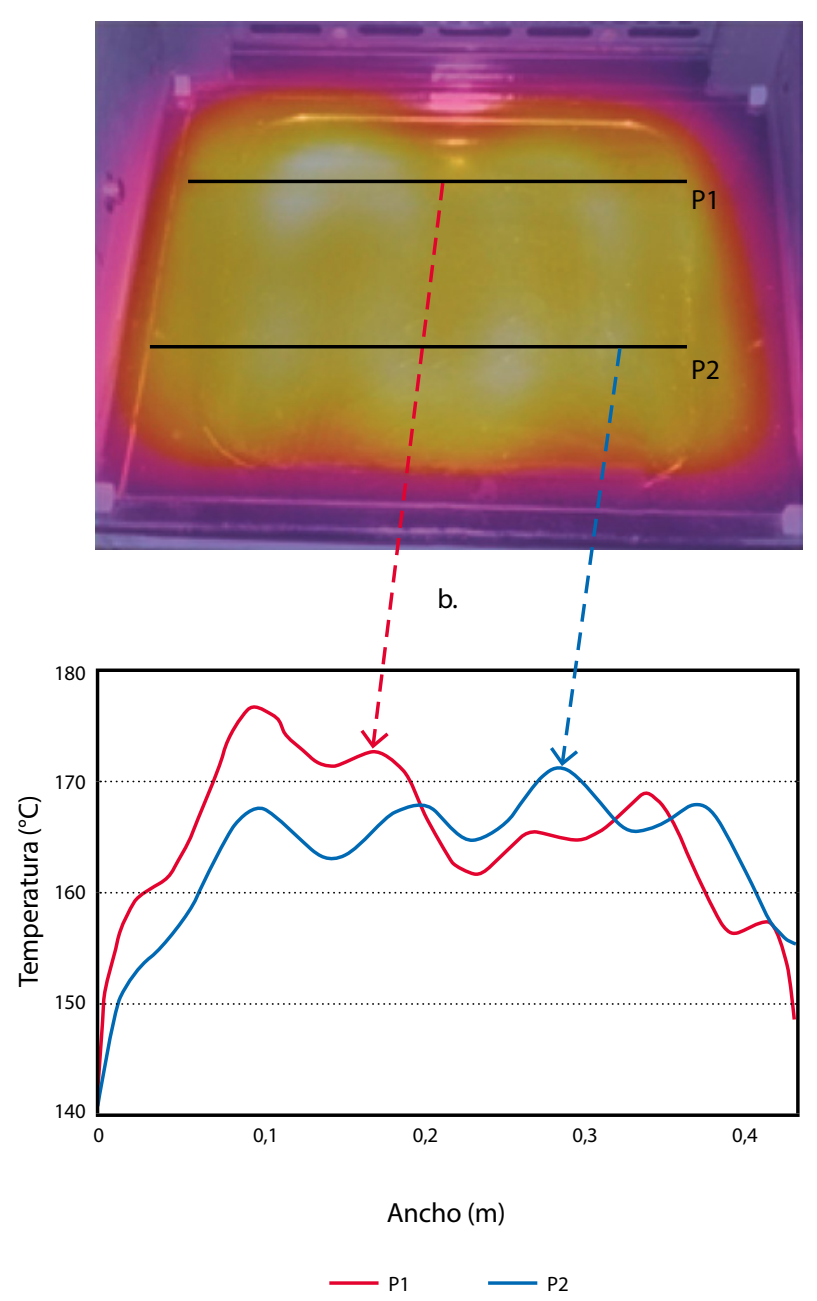

Figura 5. a) Superposición de imagen térmica y visible, mostrando la distribución de temperatura en el piso del horno. b) Variación de temperatura a lo largo del piso del horno en dos líneas arbitrarias (expresadas arriba).

Luego de que comienza a actuar el sistema de control del horno, la temperatura media experimental en el centro fue de $109,3{ }^{\circ} \mathrm{C}$, variando entre 103,2 y $115,4^{\circ} \mathrm{C}$, y la simulada fue de $105,75^{\circ} \mathrm{C}$, variando entre 95,39 y $115,90^{\circ} \mathrm{C}$. La temperatura simulada promedio integrada en el volumen del horno varió entre $\sim 95 \mathrm{y} \sim 114^{\circ} \mathrm{C}$, lo cual es muy cercano a la temperatura medida en el centro del horno. Las diferencias 


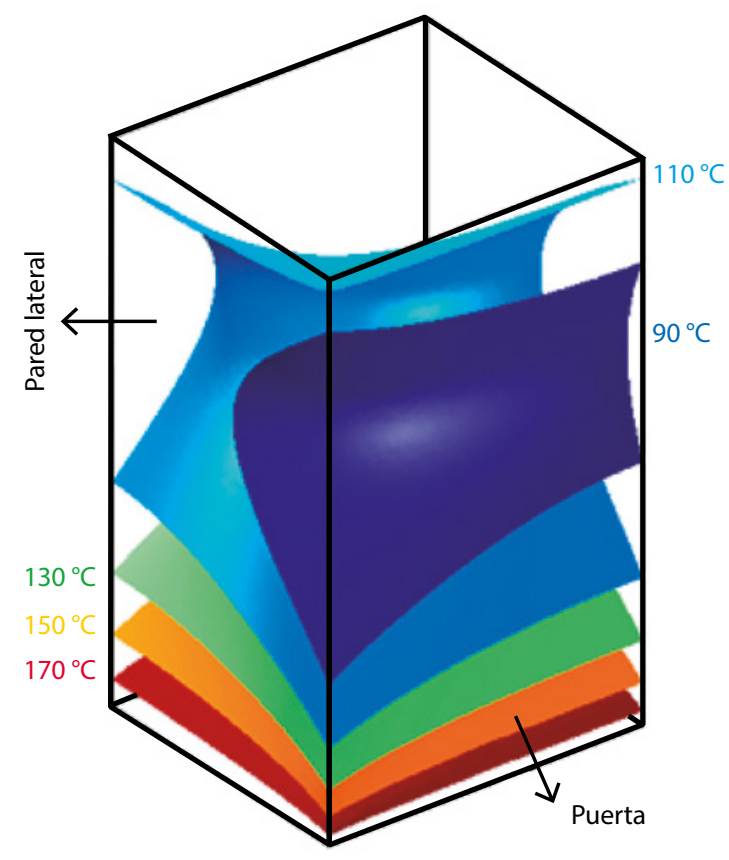

Figura 6. Perfiles de temperatura (constante) obtenidos con el modelo matemático, a 10 min de proceso.

de temperatura absoluta promedio en los distintos puntos fue de $5,8^{\circ} \mathrm{C}$ para el centro, $10,1^{\circ} \mathrm{C}$ en las cercanías de la puerta, $12,9^{\circ} \mathrm{C}$ en las cercanías de la pared y $7,2^{\circ} \mathrm{C}$ entre el centro y la pared. Las diferencias observadas entre los perfiles experimentales y simulados se deben a múltiples factores, entre ellos las propiedades térmicas y la aproximación de las fuerzas de flotación, la aproximación de las condiciones de contorno del balance de energía y la simplificación de la geometría. Se impuso la temperatura en el techo y el piso de manera uniforme, lo cual determina un flujo de energía distinto al real. Esto se debe a que no todo el piso está a la temperatura medida, sino que puede haber zonas con menor o mayor temperatura, dado que la resistencia eléctrica no es uniforme en todo el piso, como puede verse en la Figura 5. Por otro lado, en el techo se puede mejorar el modelo incorporando las resistencias a la geometría.

La Figura 6 muestra los perfiles de temperatura simulados a 10 min de proceso; el perfil de $90^{\circ} \mathrm{C}$ se encuentra más cercano a la puerta, donde hay mayores pérdidas de energía, lo cual coincide con los resultados experimentales.

La Figura 7 presenta tres planos (en los planos de simetría y a la altura media del horno) de velocidad vertical a $10 \mathrm{~min}$ de proceso; se observa que el aire asciende por el centro y desciende por el lado de las paredes laterales y la puerta. La velocidad de ascenso es más alta, probablemente debido a que esta región ocupa menos espacio.

\section{Conclusiones}

El modelo implementado resultó efectivo y relativamente rápido, considerando la complejidad del problema, para simular el comportamiento del horno simplificado. El error relativo global, medido como la diferencia entre la

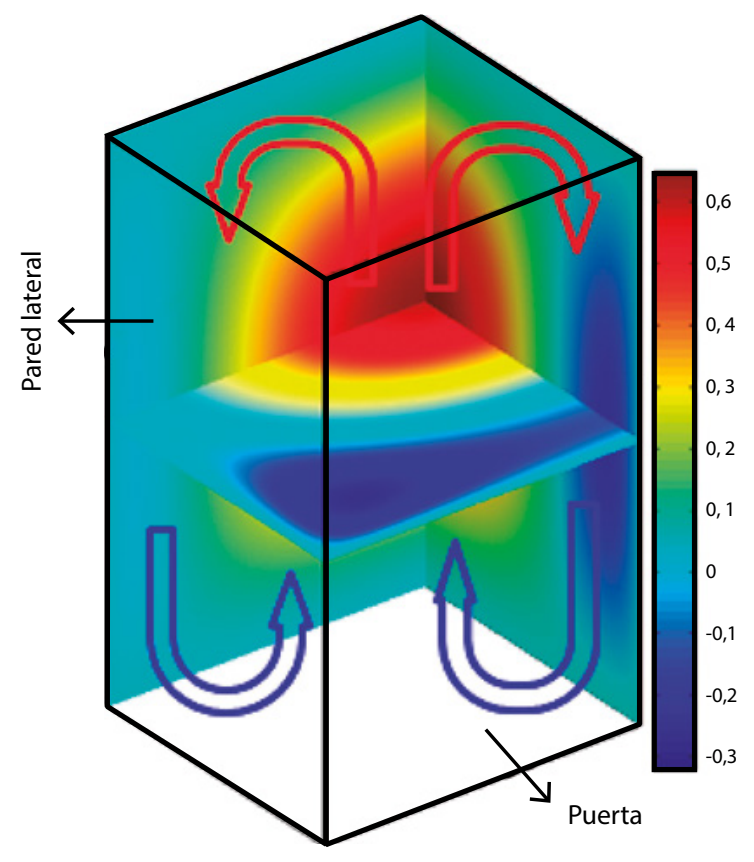

Figura 7. Perfil de velocidad vertical obtenido con el modelo, a 10 minutos de proceso.

temperatura media experimental y la simulada, respecto del valor medio experimental fue menor al $10 \%$, el cual puede considerarse un error aceptable teniendo en cuenta las suposiciones del modelo. Sin embargo, dadas las grandes variaciones de temperaturas y la variación temporal de las condiciones de contorno, el modelo laminar y la aproximación de Boussinesq están al límite de sus posibilidades para poder representar adecuadamente el perfil de velocidad y de temperatura. Asimismo, si se incorporaran más detalles del horno, como rejillas, esto puede conducir a regiones donde el modelo laminar no sea aplicable. Por este motivo se prevé en futuros trabajos incorporar el uso de modelos de turbulencia.

\section{Reconocimientos}

Este trabajo se financió con fondos provenientes del Consejo Nacional de Investigaciones Científicas y Técnicas (CONICET, PIP 11220120100180), Agencia Nacional de Promoción Científica y Tecnológica (ANPCyT PICT 2013-1637) y Universidad Nacional de La Plata (UNLP, I183).

\section{Referencias}

Ateeque, Md., Udayraj, Mishra, R. K., Chandramohan, V. P. y Talukdar, P., 2014. Numerical modeling of convective drying of food with spatially dependent transfer coefficient in a turbulent flow field. En: International Journal of Thermal Sciences, 78, pp.145-157.

Boulet, M., Marcos, B., Dostie, M. y Moresoli, C., 2010. CFD modeling of heat transfer and flow field in a bakery pilot oven. En: Journal of Food Engineering, 97(3), pp.393-402. 
Chhanwal, N., Anishaparvin, A., Indrani, D., Raghavarao, K.S.M.S. y Anandharamakrishnan, C., 2010. Computational fluid dynamics (CFD) modeling of an electrical heating oven for bread-baking process. En: Journal of Food Engineering, 100(3), pp.452-460.

Chhanwal, A., Indrani, D., Raghavarao, K.S.M.S. y Anandharamakrishnan, C., 2011. Computational fluid dynamics modeling of bread baking process. En: Food Research International, 44(4), pp.978-983.

Ferziger, J. H. y Perić, M., 2002. Computational methods for fluid dynamics. Berlin: Springer-Verlag.

Goñi, S. M. y Salvadori, V. O., 2012. Model-based multiobjective optimization of beef roasting. En: Journal of Food Engineering, 111(1), pp.92-101.

Goñi, S.M. y Salvadori, V.O., 2014. Energy consumption estimation during oven cooking of food. En: Reiter, S., ed. Energy consumption: impacts of human activity, current and future challenges, environmental and socio-economic effects. New York: Nova Science Publishers. pp.99-116.

Khatir, Z., Paton, J., Thompson H., Kapur, N. y Toropov, V. 2013. Optimisation of the energy efficiency of bread-baking ovens using a combined experimental and computational approach. En: Applied Energy, 112, pp.918-927.

Marra, F., De Bonis, M.V. y Ruocco, G., 2010. Combined microwaves and convection heating: a conjugate approach. En: Journal of Food Engineering, 97(1), pp.31-39.

Mistry, H., Ganapathi-subbu, Dey, S., Bishnoi, P., Castillo, J.L., 2006. Modeling of transient natural convection heat transfer in electric ovens. En: Applied Thermal Engineering, 26(17-18), pp.2448-2456.
Mistry, H., Ganapathisubbu, S., Dey, S., Bishnoi, P. y Castillo, J.L., 2011. A methodology to model flow-thermals inside a domestic gas oven. En: Applied Thermal Engineering, 31(1), pp.103-111.

Norton, T. y Sun, D.-W. 2006. Computational fluid dynamics (CFD) - an effective and efficient design and analysis tool for the food industry: A review. En: Trends in Food Science \& Technology, 17(20), pp. 600-620.

Paton, J., Khatir, Z., Thompson H., Kapur, N. y Toropov, V., 2013. Thermal energy management in the bread baking industry using a system modelling approach. En: Applied Thermal Engineering, 53(2), pp.340-347.

Smolka, J., Nowak, A.J. y Rybarz, D., 2010. Improved 3-D temperature uniformity in a laboratory drying oven based on experimentally validated CFD computations. En: Journal of Food Engineering, 97(3), pp.373-383.

Smolka, J. 2013. Genetic algorithm shape optimisation of a natural air circulation heating oven based on an experimentally validated 3-D CFD model. En: International Journal of Thermal Sciences, 71, pp.128-139.

Tzempelikos, D.A., Mitrakos, D., Vouros, A.P., Bardakas A.V., Filios, A.E. y Margaris, D.P., 2015. Numerical modeling of heat and mass transfer during convective drying of cylindrical quince slices. En: Journal of Food Engineering, 156, pp.10-21.

Verboven, P., Scheerlinck, N., De Baerdemaeker, J. y Nicolaï, B.M., 2000. Computational fluid dynamics modelling and validation of the temperature distribution in a forced convection oven. En: Journal of Food Engineering, 43(2), pp.61-73. 